\title{
Casein kinase I epsilon somatic mutations found in breast cancer cause overgrowth in Drosophila
}

\author{
TOMAS DOLEZAL ${ }^{*}, 1,2$, KATERINA KUCEROVA ${ }^{1}$, JANA NEUHOLD ${ }^{1}$ and PETER J. BRYANT ${ }^{3}$ \\ ${ }^{1}$ Department of Molecular Biology, Faculty of Science, University of South Bohemia in Ceske Budejovice, \\ ${ }^{2}$ Institute of Entomology, Biology Centre of the Academy of Sciences of the Czech Republic, Czech Republic \\ and ${ }^{3}$ Developmental Biology Center, University of California, Irvine, USA
}

\begin{abstract}
We are using a candidate gene approach to identify genes contributing to cancer through somatic mutation. Somatic mutations were found in breast cancer samples in the human casein kinase I epsilon (CKIE) gene, a homolog of the Drosophila gene dco in which certain point mutations lead to imaginal disc overgrowth. We therefore created fly genotypes in which the dco gene carried point mutations homologous to those discovered in $C K I \varepsilon$, and tested them in vivo. The results show that the most frequent mutation discovered in breast cancer, L39Q, causes a striking overgrowth phenotype in flies. Further experiments show that this mutation affects the newly recognized Fat/Warts signaling pathway, which controls organ size and shape in both flies and mammals. Another mutation, S101R, modifies the mutant phenotype so that the affected tissue disintegrates, mimicking more aggressive forms of breast cancer. Our results thus strongly support the conclusion that $C K I \varepsilon$ mutations play important roles in breast carcinogenesis.
\end{abstract}

KEY WORDS: Drosophila, breast cancer, CKIE, Dco

\section{Introduction}

Most of the genes known to be causally related to human cancer have been identified through their association with familial cancers, but this approach identifies only a very limited set of cancer-related genes. Strictly somatic mutations are probably extremely important in all stages of carcinogenesis but are not well characterized because of their probable heterogeneity and the lack of practically feasible methods to identify the affected genes. However, candidate genes that may be affected by somatic mutations can potentially be identified by their homology to genetically characterized cancer-related genes in model organisms, specifically mouse and Drosophila. Tests in model systems also provide the opportunity to distinguish functionally significant somatic mutations from bystander mutations that do not contribute to the malignant phenotype (Zender et al. 2006).

Fuja et al. (2004) screened breast cancer tissue for somatic mutations in candidate genes identified through their homology to tumor suppressor genes where mutations cause either hyperplastic or neoplastic overgrowth of the imaginal discs in larval Drosophila. They found a remarkably high rate of mutations in the human gene encoding Casein Kinase $I \varepsilon(C K I \varepsilon)$, homologous to the Drosophila disc overgrown (dco) gene in which an unusual allele $d c o^{3}$ causes imaginal disc overgrowth (Jursnich et al. 1990; Zilian et al. 1999). All of the 11 identified human mutations were non-synonymous, ten of them altered residues conserved from Drosophila to human (Fig. 1B), and most of them were associated with loss of heterozygosity of closely linked markers, all indicating that these mutations were functionally significant and contributed to the cancer phenotype. One particular mutation, L39Q, was found in 5 out of 6 mutated samples, or $12 \%$ of 42 screened breast cancer patients. Although these are all indications that the identified mutations might be related to the process of carcinogenesis, a more functional study is required to test their real significance. In order to test for the predicted functional changes in vivo, we are recreating some of the identified human $C K I \varepsilon$ mutations in the highly conserved fly homolog $d c o$.

Abbreviations used in this paper:CKI, casein kinase Iepsilon; Dco, disc overgrown.

\footnotetext{
*Address correspondence to: Tomas Dolezal. Department of Molecular Biology, Faculty of Science, University of South Bohemia, Branisovska 31 Ceske Budejovice 37005, Czech Republic. Fax: +42-038-777-2265. e-mail: tomas.dolezal@prf.jcu.cz - web: http://apendix.prf.jcu.cz/Dolezal/dolezal/
}

ISSN: Online 1696-3547, Print 0214-6282 


\section{Results}

Since overexpression or misexpression of an introduced gene construct could mask the effects of a mutation, we have introduced the mutations into a $d c o$ gene regulated by adjacent genomic regulatory sequences, using a large genomic clone containing the dco gene (Fig. 1A). This clone bears intact endogenous regulatory sequences surrounding the dco gene, demonstrated by the fact that when transgenically provided to flies it fully rescued the null dco mutants (Zilian et al. 1999). We have introduced selected human mutations into this clone by site-directed mutagenesis and injected the resulting clones into embryos to establish transgenic lines. We have introduced the mutant transgenes into a null dco background (achieved by combining the deletions $d c o^{l e 88}$ and $D f(3 R) A 177 d e r 22 ;$ Fig. $1 \mathrm{~A}$ and Zilian et al.1999) so the only Dco protein present was the mutant version expressed from the natural promoter. For L39Q, we will refer to this genetic combination simply as the $d c 0^{L 39 Q}$ mutant.

The $d c o^{L 39 Q}$ mutant causes an extended larval development (10 days compared to 5 days for wild-type larvae) associated with a striking overgrowth phenotype in larval imaginal discs (Fig. 2). Whereas the wild-type discs stop growing when they reach the appropriate size (as in Fig. 2A), the mutant discs continue growing during the extended larval period and before pupariation they reach about three times the normal final size. Although the mutant larvae eventually pupate they do not proceed through metamorphosis, and the pupae die without any signs of adult development.

The effect of $d c o^{L 39 Q}$ on larval development and disc growth is similar to the phenotype of the originally isolated $d c o^{3}$ mutant (compare discs in Fig. 2A), but the overall morphology of $d{ }^{2} O^{L 39 Q}$ discs is much more abnormal, with more extensive rippling and loss of normal morphological features including the wing pouch. These features are recessive towards $d c o^{3}$, since $d c o^{L 39 Q} / d_{c o} o^{3}$ transheterozygotes appear similar to $d_{c o^{3}}$. In spite of this overgrowth, confocal microscopy revealed that the $d c O^{L 39 Q}$ mutant discs retain their monolayered epithelial structure (Fig. 3) showing that they exhibit a hyperplastic overgrowth phenotype as is typical for $d c o^{3}$ and other mutants in the Fat/ Warts cascade (Bryant et al. 1988; Jursnich et al. 1990; Justice et al. 1995; Xu et al. 1995; Cho et al. 2006).

In breast cancer samples the $C K I \varepsilon$ L39Q mutation was usually observed in combination with additional mutations in the same gene (Fig. 1 and Fuja et al. 2004); for example, in two breast cancer patients L39Q was associated with another point mutation S101R. Therefore we tested this combination of mutations in flies, and found that $d c 0^{L 39 Q, S 101 R}$ mutants have extended larval development and a similar imaginal disc morphology to that seen in $d c o^{L 39 Q}$ when they reach the size of wildtype discs (Fig. 2A). However, the surface of $d c 0^{L 39 Q, S 101 R}$ mutant discs is not smooth as in wild type or $d c o^{L 39 Q}$, the discs do not grow larger than normal, and the tissue seems to disintegrate in places (Fig. $2 \mathrm{~A}$ and in more detail in Fig. 2E). We conclude that the growth of $d c O^{L 39 Q, S 101 R}$ discs is affected as in $d c o^{L 39 Q}$ but that in addition the S101R mutation causes loss of tissue integrity. It is important to note that, although in both human cancer cases S101R was found in combination with L39Q, a heteroallelic combination could not be excluded. Therefore it will be interesting to test, using our Drosophila model, the effects of individual S101R mutation as well as other combinations of identified somatic mutations.

For both mutants, $d c o^{L 39 Q}$ and $d c o^{L 39 Q, S 101 R}$, two independent transgenic lines gave indistinguishable mutant phenotypes, making it unlikely that the phenotypes are caused by changed expression of dco due to a position effect on the transgenic insertion.

The $d c o^{L 39 Q}$ mutant phenotype closely resembles that produced by mutants in the Fat/Warts signaling pathway (Cho et al. 2006 and Fig. 4A), namely overgrowth and rippled morphology of the imaginal discs (Justice et al. 1995; Bennett \& Harvey, 2006). Furthermore, in two recent studies it was shown that the Dco kinase phosphorylates the Fat receptor (Feng et al. 2009; Sopko et al. 2009). Therefore, we are testing whether $d c^{L 39 Q}$ also affects Fat/Warts signaling. To do this we have taken advantage of the fact that the $d c 0^{L 39 Q}$ mutation expresses with low penetrance (less than 15\%) a very mild dominant effect on a crossvein in the adult wing (Fig. 4C). As shown in Fig. 4C,

A

A $\operatorname{Sox} 100 B$
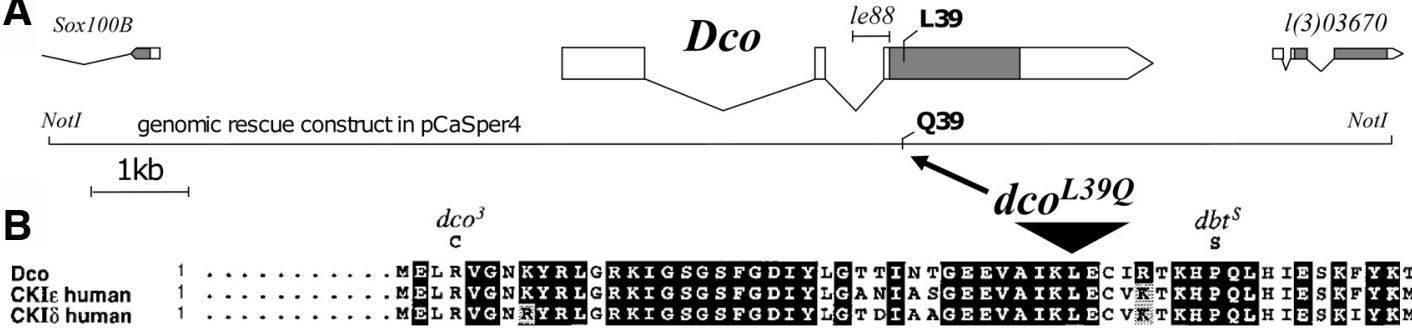

$1 \mathrm{~kb}$

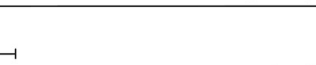

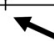

(1)
mutagenesis and mutations in dco and CKIE. (A) Schematic map of the genomic region containing the dco gene; exons are depicted by boxes with protein-coding region in grey. The position of leucine L39 which was mutated to glutamine 039 is shown. The small 391-bp deletion le88, which removes the start codon of dco, was used with the Df(3R)A177der22 deficiency (covering a larger area than on the map) to make a null dco mutant background. The size and position of the genomic rescue construct (Zilian et al. 1999) in which the mutations were introduced is also shown.

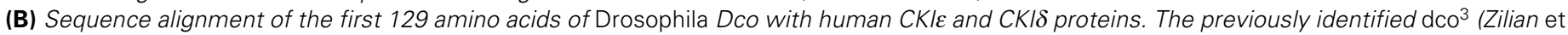
al. 1999) doubletime ${ }^{\mathrm{L} / \mathrm{S}}$ (Kloss et al. 1998) and newly introduced dco $\mathrm{L390,S101 \textrm {R }}$ mutations are shown above the alignment. Somatic mutations found in human CKIE in breast cancer are shown below the alignment with the number of their occurrences in parentheses. 


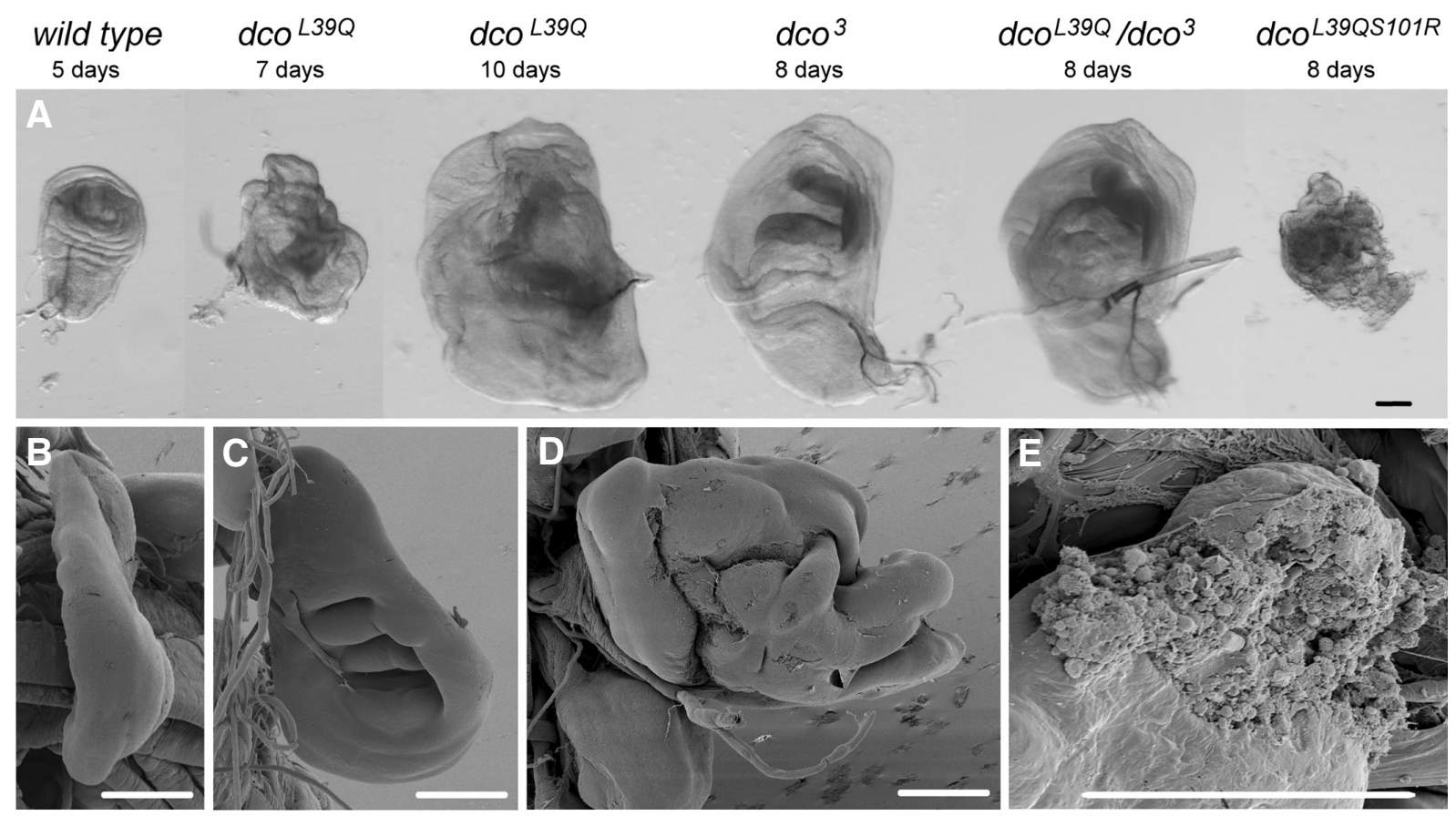

Fig. 2. Wing disc morphology of different dco mutants. (A) Wing discs dissected from third-instar larvae of various genotypes indicated above each disc; the age of larvae in days after egg laying is also indicated. (B-E) Scanning electron microscopy of wing discs. Side view showing flat plane (B) and bottom view showing typical morphology of late third-instar wild-type wing disc (C). Slant view of dco ${ }^{390}$ mutant disc demonstrating overgrowth of tissue in all directions (D). Detail of ruptured tissue of dco ${ }^{\mathrm{L390}, \mathrm{S} 101 \mathrm{R}}$ mutant disc (E). Scale bar on all pictures, $100 \mu \mathrm{m}$

while warts $^{P 2} /+$ adult wings (Justice et al. 1995) do not show any abnormalities in vein pattern, warts $^{P 2}$ significantly enhances the mild dominant effect of $d c O^{L 39 Q}$ : the extra vein fragment is present in more than $90 \%$ of the $d c o^{L 39 Q} /$ warts $^{P 2}$ transheterozygous wings, the fragment is usually enlarged, and most of the wings show additional extra vein material not present in either heterozygous combination (Fig. 4C). More importantly, the heterozygous $d c o^{L 39 Q}$ mutation significantly enlarges adult wings compared to controls (Fig. 4B and Table 1). This is similar to the effect of mutation in Warts (Fig. 4B and Table 1), which plays a central role in the control of organ size by the Fat/Warts signaling cascade. The transheterozygous $d_{c o}{ }^{L 39 Q} /$ warts $^{P 2}$ combination therefore shows a significantly
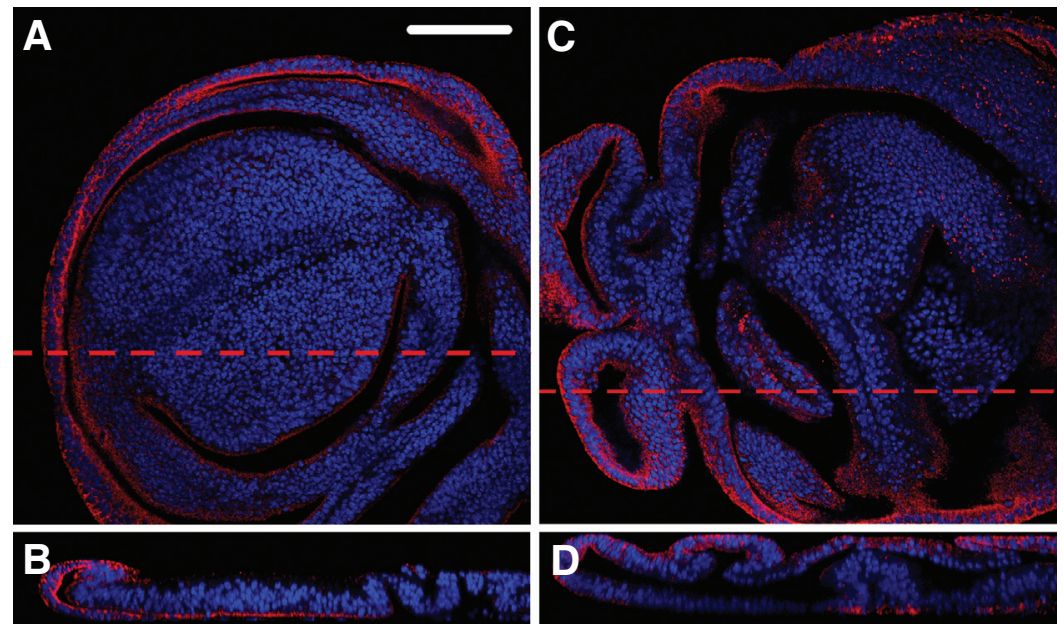

enhancement over the effects of the individual mutations (Fig. 4B and Table 1).

\section{Discussion}

This work presents a functional study of the $C K I \varepsilon$ point mutations, which were found as somatic mutations in human breast cancer, using Drosophila as a model. The introduction of the $C K I \varepsilon$ point mutation $\mathrm{L} 39 \mathrm{Q}$ into the highly conserved fly homolog dco causes a striking overgrowth phenotype in imaginal disc tissue. The newly discovered phosphorylation of the Fat receptor by Dco (Feng et al. 2009; Sopko et al. 2009), the similarity of the $d c o^{L 39 Q}$ mutant phenotype to that produced by mutants in Fat/Warts cascade, and the genetic interaction of $d c o^{L 39 Q}$ with warts $^{P 2}$ all support the idea that the $d c o^{L 39 Q}$ mutation affects the Fat/Warts signaling cascade (Fig 4A), possibly by causing abnormal phosphorylation of the Fat receptor.

The fat, discs overgrown and warts genes were discovered many years ago by their similar mutant phenotypes of hyperplastic imaginal disc overgrowth (Bryant et al. 1988; Jursnich et al. 1990; Justice et al.

Fig. 3. Confocal images of wing imaginal discs. $(\mathbf{A}, \mathbf{B})$ Wing blade region of wild-type disc with optical cross section (B) marked by red intermittent line on (A). (C,D) part of the dco ${ }^{\mathrm{L} 39 \mathrm{O}}$ wing disc. (D) An optical section demonstrating that normal epithelial structure is retained despite extensive folding. Discs were stained by DAPI labeling nuclei (b/ue) and by anti-Discs large antibody (red). White scale bar common for both images represents $60 \mu \mathrm{m}$. 
1995; Xu et al. 1995), and until recently there was no reason to consider them functionally related at the molecular level. However, the products of these genes have now been linked into a common pathway which has been called the Fat/Warts signaling cascade (Cho et al. 2006). This pathway controls organ size and shape through interaction of the Fat transmembrane receptor with its ligand Dachsous, leading to inhibition of the growthpromoting transcription coactivator Yorkie (in flies; YAP in mammals) via phosphorylation by Warts (in flies; Lats in mammals; reviewed by Reddy \& Irvine, 2008, see also Fig 4A). Rescue of the fly mutants by their mammalian counterparts has demonstrated the high evolutionary conservation of this pathway (Tao et al. 1999). Furthermore, components of the Fat/ Warts pathway are now being linked to human cancer, including breast cancer. Frequent loss of heterozygosity has been re-

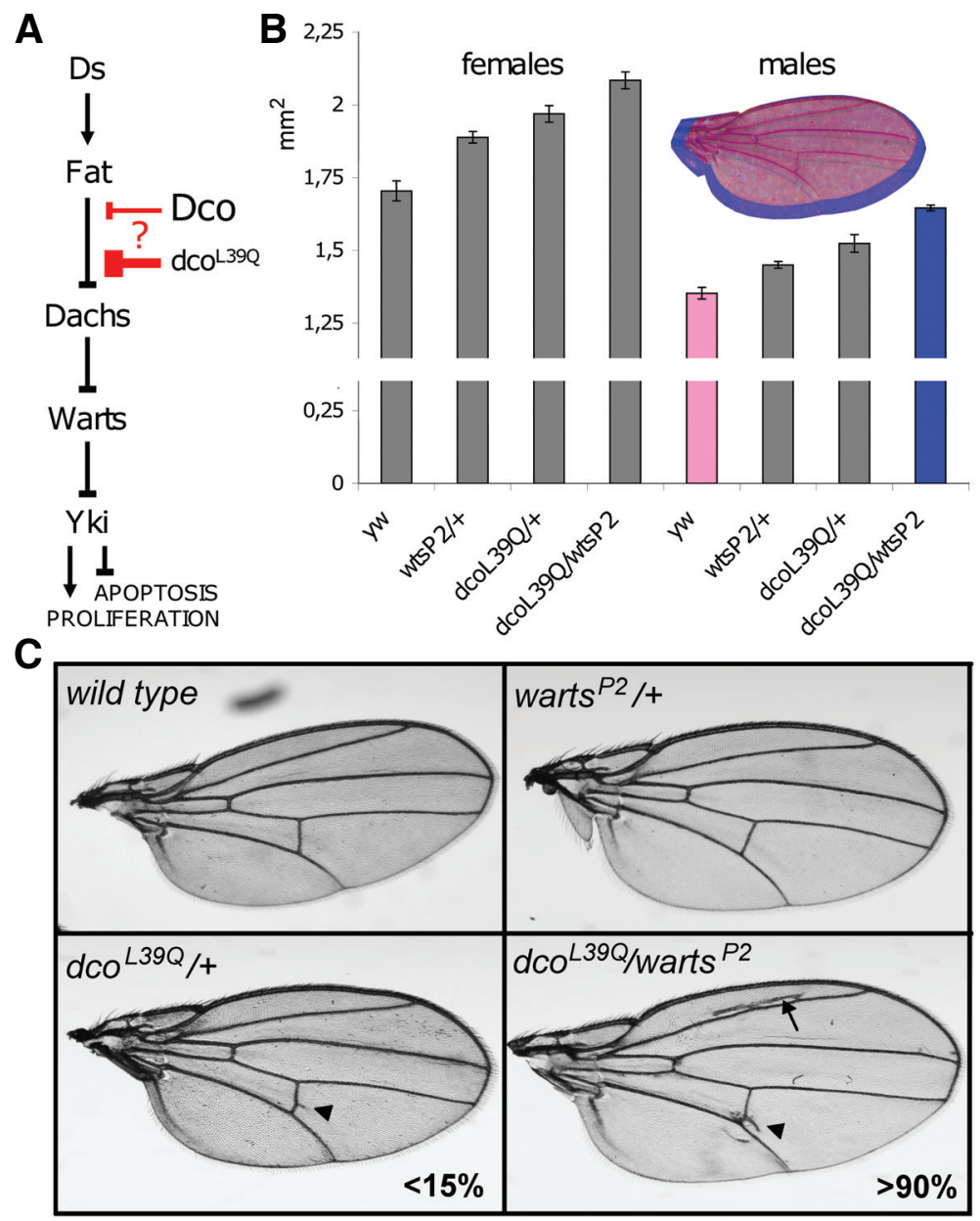

Fig. 4. Genetic interaction of $\boldsymbol{d c o}^{\mathbf{L 3 9 O}}$ with warts. (A) Simplified scheme of the Fat/Warts signaling cascade with a hypothetical role of Dco (see text for details). (B) Size of wings (in square millimeters; Mean +/-SEM) from females and males of the indicated genotypes. An image of overlapped wings of average size from yw control males (pink) and dco ${ }^{\mathrm{L} 390}$ Narts ${ }^{\mathrm{P} 2}$ transheterozygous male (b/ue) is shown within the graph for comparison. (C) Adult wings from flies of the indicated genotypes. The wing vein pattern of warts heterozygotes is normal. In less than $15 \%$ of dco ${ }^{1390}$ heterozygotes, a small branch from the crossvein (arrowhead) is detected, and a similar feature is detected in more than 90\% of $\mathrm{dco}^{\mathrm{L} 39 \mathrm{O}}$ /warts $^{\mathrm{P} 2}$ transheterozygotes. The arrow points to extra vein material which is also detected in most of the wings of the transheterozygotes.
TABLE 1

\section{COMPARISON OF WING SIZES BY TWO-SAMPLE T-TEST}

\begin{tabular}{lll} 
Compared genotypes & Females & Males \\
\hline$y w$ vs. $d c o^{L 39 Q} /+$ & $\mathrm{P}<1,01 \times 10^{-08}$ & $\mathrm{P}<1,64 \times 10^{-06}$ \\
$y w$ vs. $w t s^{P 2} /+$ & $\mathrm{P}<1,38 \times 10^{-05}$ & $\mathrm{P}<2,94 \times 10^{-06}$ \\
$d c o^{L 39 Q} /+$ vs. $d c 0^{L 39 Q} / w t s^{P 2}$ & $\mathrm{P}<4,01 \times 10^{-06}$ & $\mathrm{P}<1,71 \times 10^{-08}$ \\
$w_{t s}^{P 2} /+$ vs. $d c o^{L 39 Q} / w^{P 2}$ & $\mathrm{P}<1,76 \times 10^{-08}$ & $\mathrm{P}<1,45 \times 10^{-18}$ \\
\hline
\end{tabular}

ported for regions containing LATS1 and LATS2, the human homologs of Drosophila Warts, and promoter hypermethylation that silences these genes was detected in more than $50 \%$ of breast cancers (Takahashi et al. 2005). In a screen for gene copy-number changes in mouse mammary tumors, Overholtzer et al. (2006) identified an amplicon containing YAP, a mammalian homolog of Drosophila Yorkie and they further showed that in nontransformed mammary epithelial cells, overexpression of human $Y A P$ induces epithelialto-mesenchymal transition. Finally, Qi et al. (2009) reported loss of mRNA expression of Fat4, a human homolog of Drosophila Fat, in 3 of 5 primary breast cancers and 3 of 6 breast-cancer cell lines. Thus alterations at all levels of the Fat/Warts signaling cascade, from receptor to transcriptional coactivator, are now being reported at high frequencies for breast cancer, suggesting that aberrations of this pathway might play a significant role in breast carcinogenesis. This is strongly supported by the results presented in this paper, in that the most frequent somatic mutation of CKIE found in breast cancer causes a phenotype associated with aberrant Fat/Warts signaling in flies.

$\mathrm{Dco} / \mathrm{CK} \varepsilon$ mutations show pleiotropic effects, and different point mutations have distinct effects on Dco function. For example the $\mathrm{Dco}^{3}$ mutant protein phosphorylates the circadian rhythm protein Period normally but shows altered phosphorylation of the Fat receptor, while Dco ${ }^{\text {Dbt-AR }}$ shows altered Period phosphorylation (Feng and Irvine 2009). The normal function of Dco must be required for tissue growth, since the null mutant larvae are discless due to the reduction of apoptosis inhibitor DIAP1 (Guan et al. 2007). One possible mechanism could be that Dco fine-tunes the Fat/Warts inhibitory pathway via Fat phosphorylation (Fig. 4A). Null dco mutants, which lack this function, exhibit a reduction of DIAP1 (Guan et al. 2007), one of the Fat/Warts signaling targets. Furthermore, $d c o^{L 39 Q}$ like $d c o^{3}$ could then lead to excessive inhibition of Fat/Warts signaling, via aberrant Fat phosphorylation, resulting in excessive tissue growth (Fig. 4A).

In conclusion, our work demonstrates that introduction of the CKIE point mutation L39Q, which was found as a somatic mutation in human breast cancer, into the highly conserved fly homolog dco causes a striking overgrowth phenotype in imaginal disc tissue. This first functional study of CKIE mutations in vivo therefore strongly suggests that they indeed play an important role in breast cancer. In addition, similar phenotypes and genetic interactions indicate that $\mathrm{L} 39 \mathrm{Q}$ interacts with the recently 
recognized Fat/Warts signaling pathway, which is required for the cessation of cell proliferation when imaginal discs reach their genetically determined final size at the end of larval development. Thus our results support the idea that genes controlling this tissue-level mechanism of size control, in addition to genes controlling the basic mechanisms of cell proliferation and death, play an important role in cancer (Leevers \& McNeill, 2005). Adding the S101R mutation worsens the mutant phenotype such that the disc tissue disintegrates, which is especially interesting in view of reports that an aggressive form of breast cancer, including metastases, is associated with aberrant LATS/Yap signaling (Takahashi et al. 2005; Overholtzer et al. 2006). Our work confirms that Drosophila can be used as an extremely useful model system to identify genes that might be important in cancer (Brumby \& Richardson, 2005), and furthermore that it can provide important in vivo functional tests to characterize the importance of somatic mutations that have been discovered in human cancer.

\section{Materials and Methods}

\section{Generation of mutant flies}

Mutations L39Q and S101R were introduced into a genomic rescue construct "Notl of lambda88.9 in CasPer4\#7" (described in Zilian et al. 1999) as follows: 6-kb fragment including dco coding sequence was cut out by Xhol and BamHI and cloned into pBluescript II SK(+) (Stratagene). Mutations were introduced by QuickChange Multi Site-Directed Mutagenesis Kit (Stratagene) using following primers:

L39Q - gtggccatcaagcAggagtgcatccgc and

S101R - cttttgttcacgccgctttCGgttgaagacggttctgct (mismatches capitalized). Mutant sequences were cloned back to genomic rescue construct using Swal and BsiWI enzymes. The mutated constructs were injected into the $y w$ embryos using a standard P-element transformation protocol. Two independent transgenic lines for each mutant construct on $\mathrm{X}$ chromosome were crossed with $y w$; dcole88/TM6B and $y w$; $D f(3 R) A 177 d e r 22 /$ TM6B lines (provided by Markus Noll) to obtain yw dco L39,/S101R; $d c o^{\text {le88/ }}$ $D f(3 R) A 177$ der22 larvae with $d c o^{L 39 Q, S 101 R}$ in dconull background. $d c 0^{L 39 Q}$ mutated sequence was verified by sequencing of $P C R$ products amplified

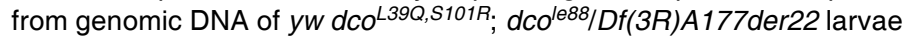
- the only normal-length PCR product was from the $d c o^{L 39 Q, S 101 R}$ sequence.

\section{Genetic interaction with warts}

$y$ w dco ${ }^{L 39 Q}$; $d c 0^{\text {le88/TM6B }}$ and $w$; warts ${ }^{P 2} / T M 6 B^{\prime}$ lines were crossed either to $y$ wor to each other to obtain $y w d c o^{L 39 Q} ; d c o^{l e 88 /+}$ or $y w$; warts ${ }^{P 2 /}$ + heterozygous flies (depicted as $d c o^{L 39 Q /+}$ or warts ${ }^{P 2} /+$ in the text) and y $w$ dco ${ }^{L 39 Q} / w$; dco $O^{l e 88}$ warts $^{+} /$dco $^{+}$warts $^{P 2}$ transheterozygous flies (depicted as $d c o^{L 39 Q} /$ warts $^{P 2}$ in the text). Adult wings were photographed on a stereomicroscope, with transmitted light for the wing veins pattern. A fixed zoom and camera resolution were used for wing area measurement, with the area of each wing being marked using the selection tool in the GNU Image Manipulation Program 2.6 and the number of pixels being determined using the histogram tool. The pixel number was then converted to square millimeters. A t-Test was used to compare the areas in different genotypes. Wings from more than 40 flies were analyzed for each genotype.

\section{Imaginal disc preparation and analysis}

Wing imaginal discs were dissected from third-instar larvae of the indicated genotype and age, and their size and morphology observed using a stereomicroscope using transmitted light. The epithelial architecture was analyzed using confocal microscopy; discs were fixed in $4 \%$ paraformaldehyde and stained using mouse anti-Disc large antibody diluted 1:400 (the mouse monoclonal antibody developed by Dr. Dan
Woods was obtained from the Developmental Studies Hybridoma Bank developed under the auspices of the NICHD and maintained by The University of lowa, Department of Biology, lowa City, IA 52242) and by DAPI.

\section{Scanning electron microscopy}

Larval bodies were inverted in PBS on ice and the fat body, gut and salivary glands were dissected away. Imaginal discs attached to the integument were fixed in glutaraldehyde, dehydrated and dried, coated by gold and scanned using a JEOL JSM-7401F scanning electron microscope.

\section{Acknowledgements}

We thank Markus Noll for providing us with dco flies and the genomic rescue construct, Eugen Kubala for help with embryo injection, Petra Masarova and team from Laboratory of electron microscopy at Biology Center of ASCR for preparing and processing of our samples. Work was funded by the Grant Agency of the Czech Republic under grant 301/07/ 0814 to T.D. and by the Ministry of Education, Youth and Sports of the Czech Republic under grant MSM6007665801.

\section{References}

BENNETT FC, HARVEY KF (2006). Fat cadherin modulates organ size in Drosophila via the Salvador/Warts/Hippo signaling pathway. Curr Biol 16: 2101-2110.

BRUMBY AM, RICHARDSON HE (2005). Using Drosophila Melanogaster to map human cancer pathways. Nat Rev Cancer 5: 626-639.

BRYANT PJ, HUETTNER B, HELD LI JR, RYERSE J and SZIDONYA J (1988). Mutations at the fat locus interfere with cell proliferation control and epithelial morphogenesis in Drosophila. Dev Biol 129: 541-554.

CHO E, FENG Y, RAUSKOLB C, MAITRA S, FEHON R and IRVINE KD (2006). Delineation of a Fat tumor suppressor pathway. Nat Genet 38: 1142-1150.

FENG Y, IRVINE KD (2009). Processing and phosphorylation of the Fat receptor. Proc Natl Acad Sci USA 106: 11989-11994.

FUJA TJ, LIN F, OSANN KE and BRYANT PJ (2004). Somatic mutations and altered expression of the candidate tumor suppressors CSNK1 epsilon, DLG1, and EDD/hHYD in mammary ductal carcinoma. Cancer Res 64: 942-951.

GUAN J, LI H, ROGULJA A, AXELROD JD and CADIGAN KM (2007). The Drosophila casein kinase lepsilon/delta Discs overgrown promotes cell survival via activation of DIAP1 expression. Dev Biol 303: 16-28.

JURSNICH VA, FRASER SE, HELD LI JR, RYERSE J and BRYANT PJ (1990). Defective gap-junctional communication associated with imaginal disc overgrowth and degeneration caused by mutations of the dco gene in Drosophila. Dev Biol 140: 413-429.

JUSTICE RW, ZILIAN O, WOODS DF, NOLL M and BRYANT PJ (1995). The Drosophila tumor suppressor gene warts encodes a homolog of human myotonic dystrophy kinase and is required for the control of cell shape and proliferation. Genes Dev 9: 534-546.

KLOSS B, PRICE JL, SAEZ L, BLAU J, ROTHENFLUH A, WESLEY CS and YOUNG MW (1998). The Drosophila clock gene double-time encodes a protein closely related to human casein kinase lepsilon. Cell 94: 97-107.

LEEVERS SJ, MCNEILL H (2005). Controlling the size of organs and organisms. Curr Opin Cell Biol 17: 604-609.

OVERHOLTZER M, ZHANG J, SMOLEN GA, MUIR B, LI W, SGROI DC, DENG CX, BRUGGE JS and HABER DA (2006). Transforming properties of YAP, a candidate oncogene on the chromosome 11q22 amplicon. Proc Natl Acad Sci USA 103: 12405-12410.

QI C, ZHU YT, HU L and ZHU YJ (2009). Identification of Fat4 as a candidate tumor suppressor gene in breast cancers. Int J Cancer 124: 793-798.

REDDY BV, IRVINE KD (2008). The Fat and Warts signaling pathways: new insights into their regulation, mechanism and conservation. Development 135 2827-2838.

SOPKO R, SILVA E, CLAYTON L, GARDANO L, BARRIOS-RODILES M, WRANA J, VARELAS X, ARBOUZOVA NI, SHAW S, SABURIS, MATAKATSU H, BLAIR $S$ and MCNEILL $H$ (2009). Phosphorylation of the tumor suppressor fat is 
regulated by its ligand Dachsous and the kinase discs overgrown. Curr Biol 19: 1112-1117.

TAKAHASHI Y, MIYOSHI Y, TAKAHATA C, IRAHARA N, TAGUCHI T, TAMAKI Y and NOGUCHI S (2005). Down-regulation of LATS1 and LATS2 mRNA expression by promoter hypermethylation and its association with biologically aggressive phenotype in human breast cancers. Clin Cancer Res 11: 1380-1385.

TAO W, ZHANG S, TURENCHALK GS, STEWART RA, ST JOHN MA, CHEN W and XU T (1999). Human homologue of the Drosophila Melanogaster lats tumour suppressor modulates CDC2 activity. Nat Genet 21: 177-181.

XU T, WANG W, ZHANG S, STEWART RA and YU W (1995). Identifying tumor suppressors in genetic mosaics: the Drosophila lats gene encodes a putative protein kinase. Development 121: 1053-1063.

ZENDER L, SPECTOR MS, XUE W, FLEMMING P, CORDON-CARDO C, SILKE J, FAN ST, LUK JM, WIGLER M, HANNON GJ, MU D, LUCITO R, POWERS S and LOWE SW (2006). Identification and validation of oncogenes in liver cancer using an integrative oncogenomic approach. Cell 125: 1253-1267.

ZILIAN O, FREI E, BURKE R, BRENTRUP D, GUTJAHRT, BRYANT PJ and NOLL $M$ (1999). double-time is identical to discs overgrown, which is required for cell survival, proliferation and growth arrest in Drosophila imaginal discs. Development 126: 5409-5420.

\section{Further Related Reading, published previously in the Int. J. Dev. Biol.}

See our recent Special Issue Placenta edited by Joan S. Hunt and Kent L. Thornburg at: http://www.ijdb.ehu.es/web/contents.php?vol=54\&issue=2-3

The mob as tumor suppressor (mats1) gene is required for growth control in developing zebrafish embryos Yuan Yuan, Shuo Lin, Zuoyan Zhu, Wenxia Zhang and Zhi-Chun Lai Int. J. Dev. Biol. (2009) 53: 525-533

Using fruitflies to help understand the molecular mechanisms of human hereditary diffuse gastric cancer Joana Caldeira, Paulo S. Pereira, Gianpaolo Suriano and Fernando Casares Int. J. Dev. Biol. (2009) 53: 1557-1561

The cellular and genetic bases of organ size and shape in Drosophila Antonio García-Bellido Int. J. Dev. Biol. (2009) 53: 1291-1303

Tumor invasion and metastasis: getting more basic to come closer to the patient. An interview with Lance A. Liotta Vincent Castronovo Int. J. Dev. Biol. (2004) 48: 559-562

Tumor suppressor and overgrowth suppressor genes of Drosophila melanogaster: developmental aspects E Gateff Int. J. Dev. Biol. (1994) 38: 565-590

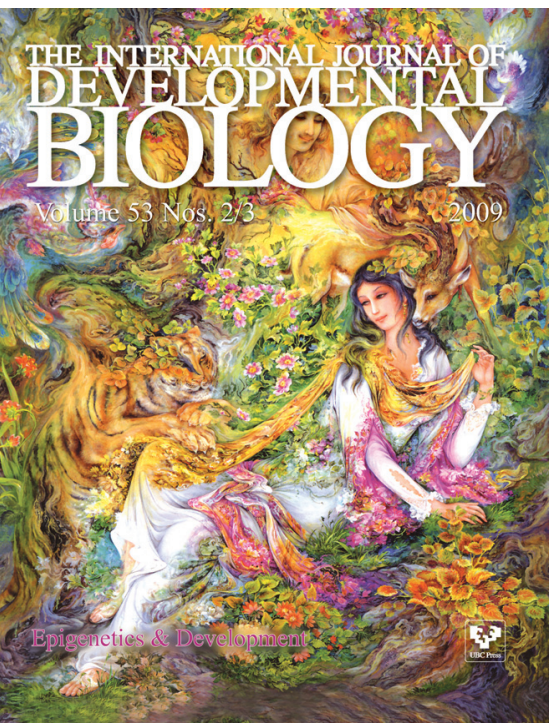

5 yr ISI Impact Factor $(2008)=3.271$

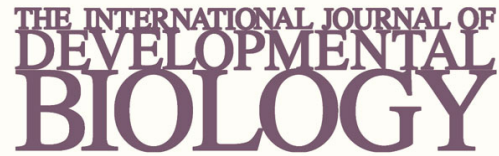

Volume 54 Nos. $6 / 7$
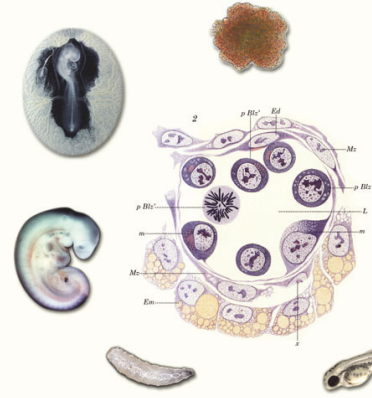

Developmental Hematopoiesis
Special Issue
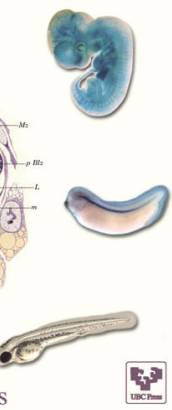

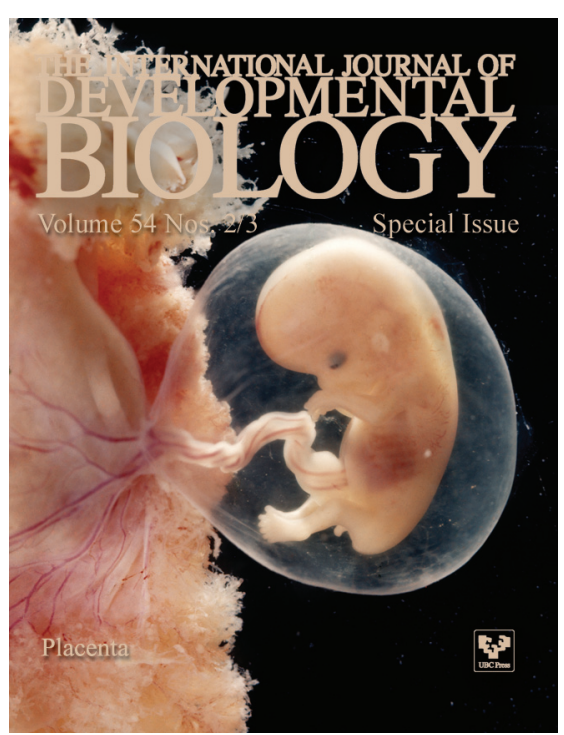

